\title{
Depth defect retrieval using the wavelet pulsed phased thermography
}

\author{
by F. Galmiche, X. Maldague \\ Université Laval, LVSN, E.E. Dep., Québec, (QC) G1K 7P4 Canada.e-mail : maldagx@gel.ulaval.ca
}

\begin{abstract}
:
Pulsed Phased Thermography (PPT) is a known method to process thermograms in pulsed thermography through a Fourier frequency analysis. However, this Fourier transform is limited to a qualitative analysis. This paper shows that the wavelet transform enables to perform both a qualitative and a quantitative analysis. Experimental and simulation results are presented as well.
\end{abstract}

\section{Introduction}

Pulsed Phased Thermography (PPT) has been introduced in 1996 [1]. This innovative method was presented as a new analysis technique for pulsed thermography (PT). The obtained results are qualitatively very interesting. They permit the easy detection of subsurface structures with enhanced visibility. However, attempts to make a quantitative analysis based on PPT showed important limitations [2,3]. This article explains the reason of such limitations. The wavelet transform is presented as a new signal processing method to bypass such limitation. Experimental and simulation results are presented.

\section{Theory}

\subsection{Pulsed phase thermography}

Pulsed phased thermography is a practical way to analyse data obtained from a PT experiment. A short pulse of energy is applied to the surface of the specimen to be analyzed using lamps or powerful photographic flashes. The temperature evolution of the surface is recorded using an infrared camera (IR). The measurement is made in the transient state. The Fourier transform of the temporal evolution of the temperature $\mathrm{T}(\mathrm{t})$ of each pixel, where $\mathrm{t}$ is the time, is calculated [4]:

$$
\text { Fourier_Transform }[\mathrm{T}(\mathrm{t})]=\mathrm{FT}(\mathrm{f})=\int_{-\infty}^{+\infty} \mathrm{T}(\mathrm{t}) \mathrm{e}^{-\mathrm{i} 2 \pi \mathrm{tf}} \mathrm{dt}=\mathrm{A}(\mathrm{f}) \mathrm{e}^{\mathrm{i} \phi(\mathrm{f})}
$$

where $F T(f)$ is the Fourier transform of $T(t), A(f)$ is the amplitude of the result and $\phi(f)$ its phase. The phase gives good qualitative results for subsurface defect detection [5].

The main drawback of this method is related to an important property of the Fourier transform [5]. In fact, the Fourier basis function has infinite extensions along the time axis. This means that the FT does not provide any information regarding the time evolution of spectral characteristics of the signal. The problem is that in PT, the time information is related to the depth of observed structures, so it is difficult to obtain quantitative information using the Fourier transform. The use of another transform is thus necessary in order to extend the PPT from a qualitative method to a quantitative method. One such tool is the wavelet transform.

\subsection{Wavelet transform}




\subsubsection{Theory}

The wavelet transform (WT) of a function $f(t)$ using the daughter wavelet $h_{S T}(t)$ is defined as:

$$
\mathrm{W}_{\mathrm{f}}(\mathrm{S}, \mathrm{T})=\int_{-\infty}^{+\infty} \mathrm{f}(\mathrm{t}) \mathrm{h}_{\mathrm{ST}}^{*}(\mathrm{t}) \mathrm{dt}
$$

where * denotes the complex conjugated [6]. The daughter wavelet $h_{S, T}(t)$ is generated by scaling and translation from a single basis wavelet $\mathrm{h}(\mathrm{t})$, called the mother wavelet:

$$
h_{S T}(t)=\frac{1}{\sqrt{S}} h\left(\frac{t-T}{S}\right)
$$

where $S$ is the scaling factor and $T$ the translation factor. The scaling factor is related to the frequency, and the translation factor $\mathrm{T}$ to the time. This means that this transform allows a frequency analysis without loosing the time information [7] needed for the depth defect retrieval.

The wavelet transform of $f(t)$ could be seen as a correlation between the signal and the scaled mother wavelet. This means that if $\mathrm{H}$ is the Fourier transform of $\mathrm{h}(\mathrm{t})$ and $\mathrm{FT}$ the Fourier transform of $f(t)$, the wavelet transform can be written as the inverse Fourier transform of their product:

$$
\mathrm{W}_{\mathrm{f}}(\mathrm{S}, \mathrm{T})=\frac{\sqrt{\mathrm{S}}}{2 \pi} \int_{-\infty}^{+\infty} \mathrm{FT}(\omega) \cdot \mathrm{H}^{*}(\omega) \exp (-\mathrm{j} \omega \mathrm{T}) \mathrm{d} \omega
$$

It is also worthwhile to mention that the time-bandwidth product must obey the Heisenberg uncertainty principle [7]:

$$
\Delta \mathrm{t} \cdot \Delta \omega \geq 1 / 2
$$

\subsubsection{Application to PPT}

The wavelet transform permits the use of different mother wavelets. Each one has specific advantages. In fact the idea of the wavelet transform is to decompose the signal of interest in scaled and translated replicas of the mother wavelet. In the case of the Fourier transform, the signal is decomposed into an infinite summation of sinusoids. Contrary to sinusoids, wavelets are of finite duration. In the particular case of PPT advantages of the Fourier transform are to be kept. This is why an interesting mother wavelet is the Morlet wavelet defined as [7]:

$$
h(t)=\exp \left(-j \omega_{0} t\right) \exp \left(-\frac{t^{2}}{2}\right)
$$

This function of is a multiplication of the Fourier basis with a Gaussian window. The Fourier base allows frequency decomposition like the FFT and the Gaussian window keeps the important time information. Figure 1 shows the FT of the Morlet wavelet, which consists of two Gaussian functions shifted to w0 and $-w 0$. This means that the scaling factor allows to examine different frequencies at a time defined by the translation factor.

For the Morlet wavelet $\Delta t \bullet \Delta \omega=1 / 2$ [7]. Each value of $W_{f}(S, t)$ is thus defined within a rectangle of $\Delta t \bullet \Delta \omega$ around $T$ and $S$. This limitation of the precision in the position imposes the choice of $\omega 0$. The larger $\omega 0$ is, the smaller $\Delta \omega$ is; then greater are the uncertainties over the translation factor $\Delta \mathrm{T}$, and thus over defect depth.

A wavelet transform decorellates a one-dimensional signal (time) in a two dimensional signal (time-scale). This property increases the memory and the time required for the associated computations. It is possible to reduce this amount of computations using two 
well-known properties of a PT experience. The first one is the approximate relationship between the depth $z$ of the defect and the time t of its appearance [8]:

$$
\mathrm{t}=\frac{\mathrm{z}^{2}}{\delta}
$$

where $\delta$ is the thermal diffusivity of the material. The second one is the thermal diffusion length $\mu$, which gives an idea of the maximum penetration depth of each frequency $\omega$ within the thermal pulse [9]:

$$
\mu=\sqrt{2 \delta / \omega}
$$

The combination of these two equations, using $z$ equal $\mu$, reduces the analyzed time and frequency to those corresponding to:

$$
\mathrm{t}=\frac{2}{\omega}
$$

The translation factor $T$ corresponds to the observation time $t$ and the scale $S$ to $\omega_{0} / \omega$. The Scale and translation factor values are limited by:

$$
\mathrm{S}=\frac{\omega_{0}}{2} \mathrm{~T}
$$

Using equation 10, it is possible to keep the dimensionality of the analysed signal by calculating one scale factor for each value of $T$. The values of $T$ are limited to the values of the time of the acquisition and thus:

$$
\mathrm{W}_{\mathrm{f}}(\mathrm{S}, \mathrm{T})=\int_{-\infty}^{+\infty} \mathrm{f}(\mathrm{t}) \frac{1}{\sqrt{0.5 \cdot \omega_{0} \cdot \mathrm{T}}} \mathrm{h}\left(\frac{\mathrm{t} / \mathrm{T}-1}{0.5 \cdot \omega_{0}}\right) \mathrm{dt}
$$

Following this analysis, the wavelet transform is applied to the pulsed thermography data. The phase of the wavelet transform is used for the defect detection. A dedicated algorithm is used for defect segmentation through a Sobel edge detector. Once defect edges are defined, the image is said to be segmented and this defines defect positions [10].

The amplitude of the wavelet transform is used for the evaluation of the depth of the defect. The translation factor that gives the maximum difference between each pixel of the defect zone and the value of a free defect zone is calculated. For each pixel of the defect zone, a time that is related to the defect depth is obtained.

This wavelet transform is applied to a couple of different samples in order to calibrate the experiment. The obtained results are used to calculate a relationship between the translation factor and the depth defect.

\section{Simulation results}

This method was first tested on a thermal simulation of academic samples. A twodimensional model for PT experiments based on the finite difference method was developed. Forty-two different cases were simulated. Each case represents a piece of aluminum $5 \mathrm{~mm}$ thick with a $5 \mathrm{~mm}$ wide hole. In each case the hole has a different depth between 0.4 and $4.5 \mathrm{~mm}$.

In order to reduce de uncertainty over the results we set $\omega_{0}=2$. Six different depth defects are used in order to calibrate the process. The obtained relation is:

$$
\mathrm{z}=-1.86 \cdot 10^{3} \mathrm{t}^{2}+19.855 \mathrm{t}-7.79 \cdot 10^{-1}
$$

where $z$ is the defect depth in $\mathrm{mm}$ and $\mathrm{t}$ the time of the maximum contrast of the amplitude of the wavelet transform.

Figure 2 shows calculated depth obtained for thirty-six different defects. The error is always below $10 \%$. 


\section{Experimental results}

\subsection{Experimental set-up}

This method was applied to different plastic samples. Each one was $2.9 \mathrm{~mm}$ thick with a circular $25 \mathrm{~mm}$ wide hole. The depth of the hole varies from $0.325 \mathrm{~mm}$ to $1.375 \mathrm{~mm}$. The experimental apparatus was composed of an infrared camera and two photographic flashes, which deliver $6.4 \mathrm{~kJ}$. The sampling rate was 14.28 images per second during 30 seconds.

\subsection{Results}

In order to reduce the uncertainty over the results $\omega_{0}$ was set to 2 . Figure 3 shows the phase image for the $1.8 \mathrm{~mm}$ depth defect. The quality of this image shows that such method retains the main advantages of the Fourier transform which was the quality of obtained images.

Six different depth defects are used in order to calibrate the process. The obtained relation is:

$$
\mathrm{z}=-1.095 .10^{-5} \mathrm{t}^{2}+2.410 .10^{-4} \mathrm{t}+1.005 .10^{-4}
$$

where $z$ is the defect depth and the time of the maximum contrast of the amplitude of the wavelet transform.

Figure 4 shows calculated depths obtained for $1 \mathrm{~mm}$ depth defect. Figure 5 presents the mean of the calculated depth versus the real depth for 6 different defects. The error is always below $10 \%$ (Figure 6 ).

\section{Conclusion}

PPT using the Fourier transform gives some interesting results. In this paper we explained why these results were qualitative and not quantitative. We also explained why the wavelet transform is a better tool for PT analysis. We showed that the wavelet transform permits a quantitative analysis. We presented a fast and relatively easy way to extract quantitative and qualitative information from a PPT experiment. We presented a simulation and some experimental results, which validated this method.

\section{Acknowledgements}

NSERC of Canada and Fonds FCAR of Québec Province are thanked for their support to this work.

\section{References}

[1] X. Maldague and S. Marinetti, "Pulse Phase Infrared Thermography", Journal of Applied Physics. , Vol. 79 No. 5, (March 1996): p. 2694-2698.

[2] X. Maldague, Y. Largouet and J-P Couturier, "A study of defect depth using neural networks in pulsed phase thermography: modeling, noise, experiments", Revue Générale de Thermique, Vol 37 No317, (September 1998): p.704-717.

[3] S. Vallerand and X. Maldague, "Defect Characterization in Pulsed Thermography: A Statistical Method Compared with Kohonen and Perceptron Neural Networks", Nondestructive Testing and Evaluation International, Vol. 33, (2000), p.307-315 .

[4] R. Bracewell, The Fourier Transform and Its Applications, McGraw-Hill. New York, 1985, p. 474. 
[5] F. Galmiche, X. Maldague, S Vallerand, J.P. Couturier, "Pulsed Phased Thermography with the wavelet transform." QNDE (Montréal), p.609-615,Vol. 19A, 2000, Am. Inst. Of Physics.

[6] G. Kaiser, A friendly guide to wavelets, Birkhauser,Boston, 1994, p.300

[7] Y. Sheng, The Transforms and Applications handbook, "Chapter 10: Wavelet Transform", CRC Press, Boca Raton, IEEE Press (1996): p 747-827.

[8] X. P. Maldague, Theory and practice of IR technology in NDT, Wiley Inter Science, NewYork, 2000, (In Press).

[9] D. Wu, G. Busse, "Lock-in thermography for nondestructive evaluation of materials" Revue Générale de Thermique, Vol 37 No317, (September 1998): p.693-703.

[10] R.C. Gonzalez, R. E. Woods, Digital image processing, Addison Wesley, Reading, Massachusetts, 1992, p.716

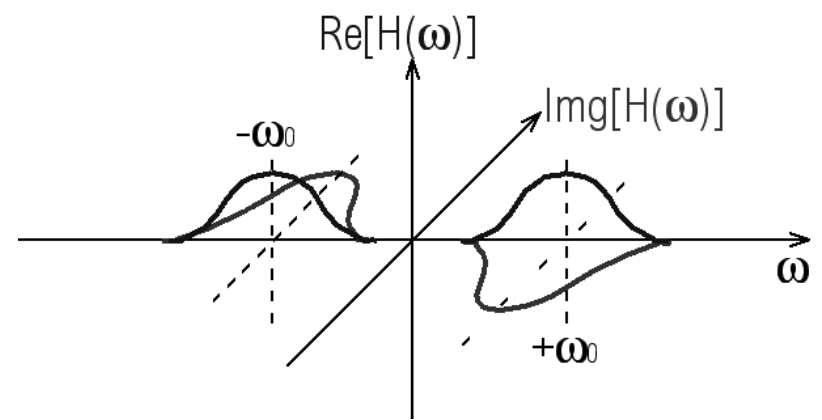

Figure1: Fourier transform of the Morlet wavelet

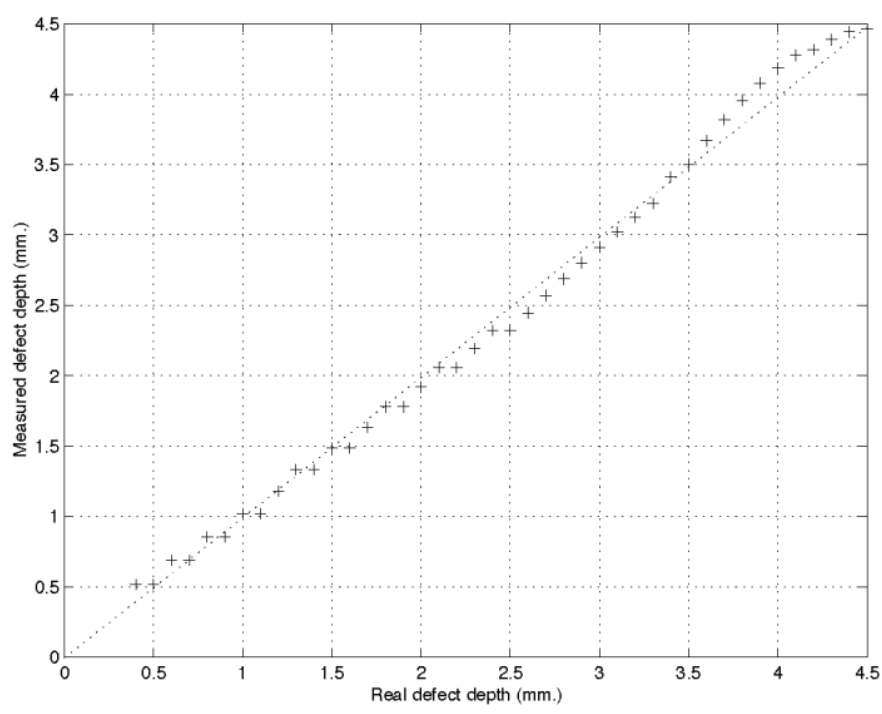

Figure 2 : Measured depth v.s. real depth for simulation samples 

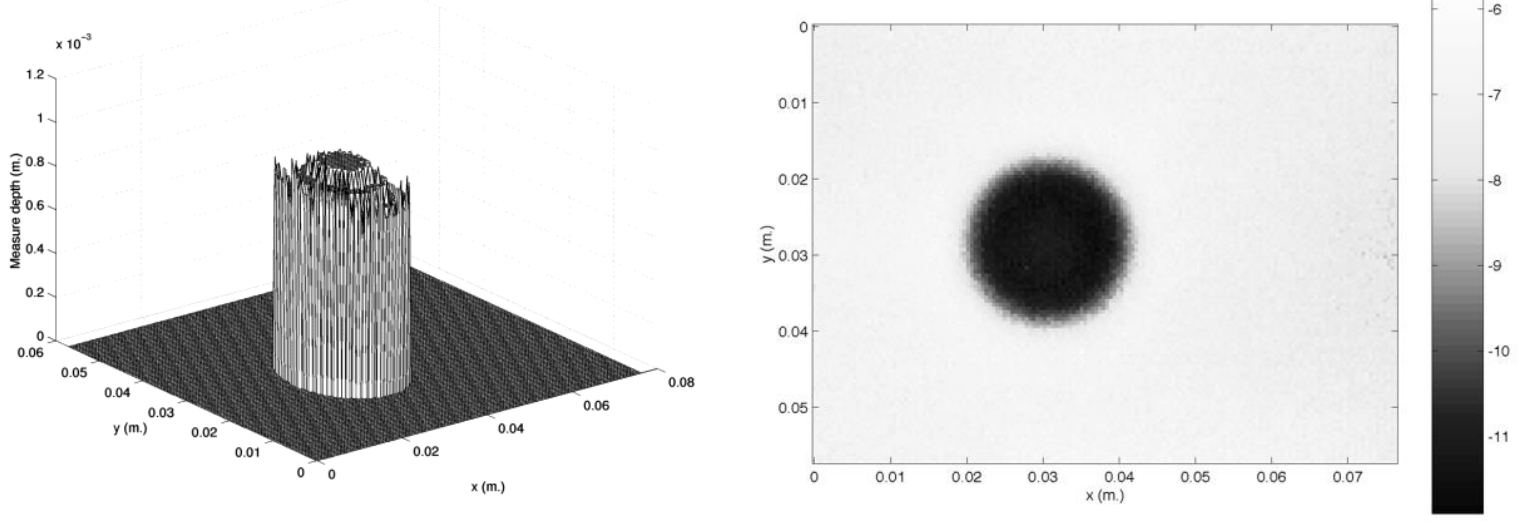

Figure 4 : Measured depth for $0.9 \mathrm{~mm}$ depth defect Figure3 : Phase image for $1.8 \mathrm{~mm}$ depth defect in plastic

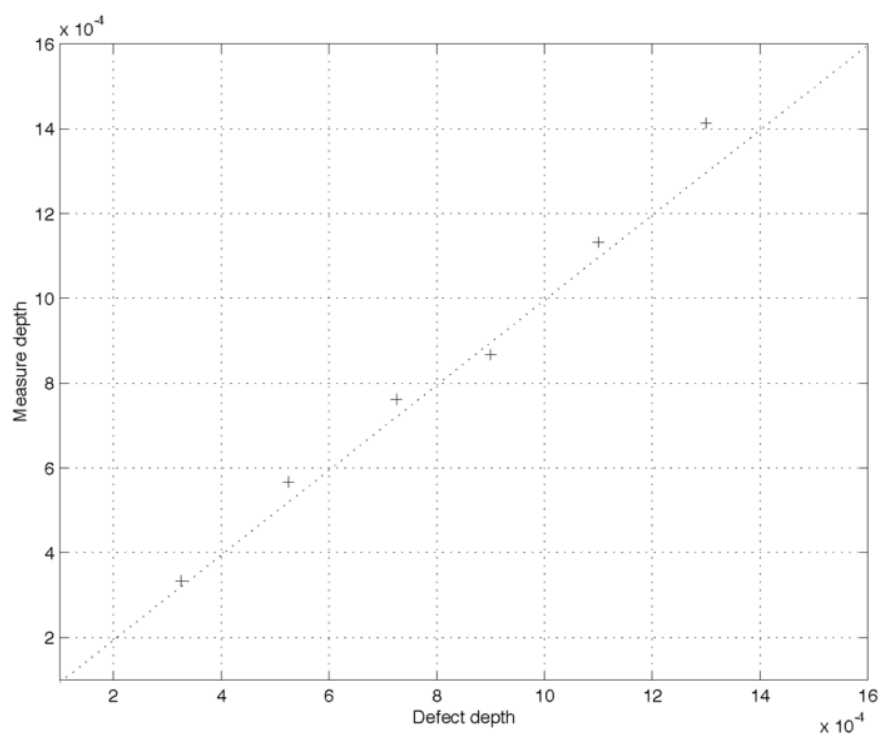

Figure 5: Measured depth v.s. real depth for experimental samples (m.)

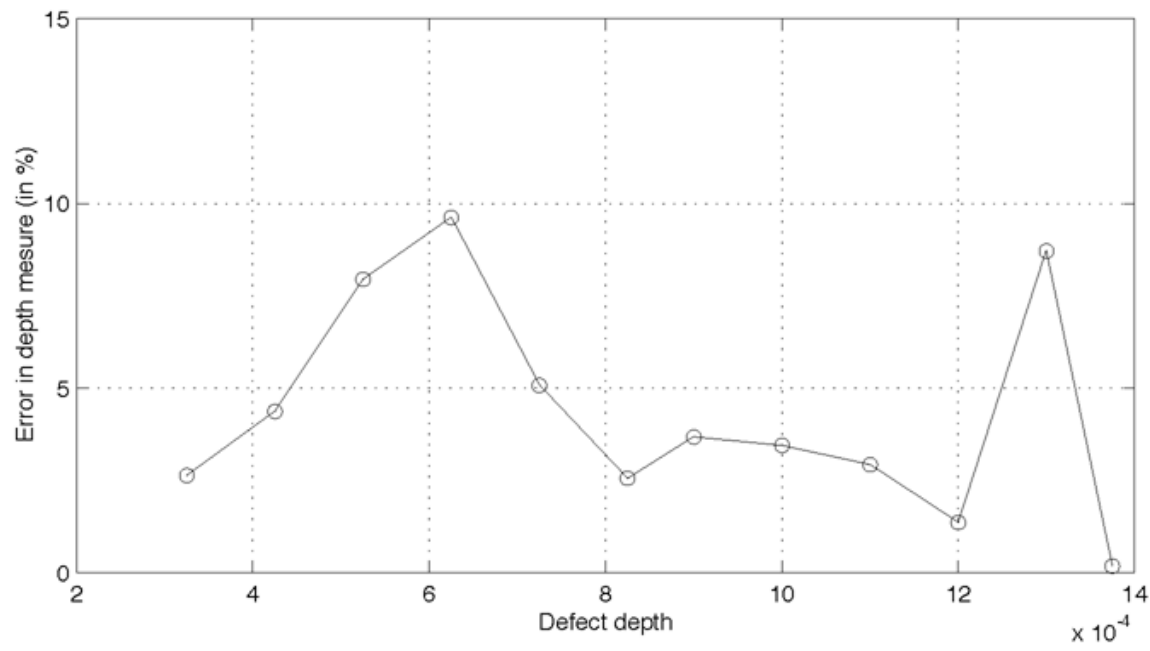

Figure 6 : Error in depth detection 